\title{
Visually controlled matching of pattern movement
}

\author{
R. WÜST, A. M. L. KAPPERS, and J. J. KOENDERINK \\ Utrecht Biophysics Research Institute, Rijksuniversiteit Utrecht, Utrecht, The Netherlands
}

\begin{abstract}
Subjects were asked to match the speeds of two moving random-dot patterns seen through circular apertures. The speed of one pattern that moved horizontally toward the right of a computer screen changed continuously. The speed of this pattern represented the target. It was to be matched with the speed of the second pattern, which moved in the opposite direction. The subject controlled the speed of the second pattern by means of an isometric joystick. The distance between the apertures on the screen as well as the subject's distance from the screen served as experimental parameters. In this way, the effects of both spatial and temporal transients of pattern speed on human tracking performance were studied. To avoid anticipation by the subject, the amplitude and the frequency of the target pattern speed changed pseudorandomly. The accuracy with which the subject performed the matching task was influenced by the mean pattern speed and the parameters of the visual field. Within lower velocity ranges, the subject's sensitivity to the instantaneous speed differences varied according to Weber's law. The cross-correlation of the velocity time courses decreased when the mean speed of the target pattern was increased. Two stimulus parameters had a strong influence on the modulation of the correlation value: (1) the angular size of the stimulus on the retina and (2) the retinal eccentricity of the stimulus.
\end{abstract}

In many situations, humans must perform visually guided navigation tasks. While steering a car or an aircraft, or just while walking around, the moving observer relies heavily on visual information, such as the distance and speed of approaching obstacles, which can be accessed through the visual image flow. This has been described most impressively by Gibson (1950). Briefly, one can say that the image flow is defined by both spatial and temporal gradients in the light distribution projected onto the retina. Psychophysical literature contains many sensitivity measurements relating to each parameter of the image flow. Precise data obtained from laboratory experiments, however, typically concern single flow parameters in isolation and are therefore not a realistic representation of the natural situation. Knowledge of an observer's sensitivity to an isolated parameter tells us something about the amount of information that can be accessed under optimum perceptual conditions. However, it does not reveal much about the importance of this information in practice. One of the major disadvantages of psychophysical experiments is that, in general, they neglect one of Gibson's most important tenets. He pointed out that

\footnotetext{
The first author is indebted to the Netherlands Organization for Scientific Research (NWO) for financial support. The coauthors acknowledge support from the InSight project of the ESPRIT programme of the European Community. We are grateful to $D$. van Touw for his participation in the experiments, to J. W. Roelofs for his essential assistance during the development of the software and to P. Faber for the development of the electronical equipment. We thank W. A. van de Grind and D. Varjú for critical reading the manuscript as well as to $S$. McNab for correcting the English language. Correspondence should be addressed to R. Wüst, who is now at Lehrstuhl für Biokybernetik, Auf der Morganstelle 28, 7400 Tübingen, Germany (e-mail: wust@fysab.fys.ruu.nl).
}

perception should not be considered as a purely passive process in the sense of simply collecting a set of stimuli that represent the external world. Nearly all percepts that humans experience in the course of a day are interactively enabled by active movements that they make. On the other hand, these movements are most often initiated and controlled by sensory information. Therefore, we regarded it as a challenge to investigate human sensitivity to optic flow parameters in experiments designed to simulate natural navigation tasks.

Using very different experimental approaches, several authors (De Bruyn \& Orban, 1988; McKee, 1981; McKee \& Nakayama, 1984; Nakayama, 1981) have described a broad range of aspects of differential motion hyperacuity, which can be quantified by a Weber ratio of around 0.05 when the stimulus has an optimum signal-to-noise ratio. Others (van Doorn \& Koenderink, 1982, 1983) have focused attention on velocity resolution, described in terms of tuning distances between hypothetical elementary movement detectors in the two-dimensional velocity space. To access the latter parameter, they made use of an experimental paradigm based on the measurement of signal-to-noise ratio thresholds for the discrimination between pattern velocities. Like other authors (McKee, 1981; Nakayama, 1981), van Doorn and Koenderink found a behavior corresponding to Weber's law. However, the sensitivity to speed transients that they measured with their technique was much lower. They found a Weber ratio ranging between $0.62 \pm 0.14$ (van Doorn \& Koenderink, 1983) and 1.0 (van Doorn \& Koenderink, 1982) for the detection of different kinds of relative movement.

Rogers and Graham (1979) showed that it is possible to extract precise depth information from movement paral- 
laxes. They found a high sensitivity (around $2 \%$ ) to slight velocity differences between moving dot patterns whose speeds were modulated by small variations in depth. The extraction of depth from movement parallaxes is one of the main perceptual problems that have to be solved in navigation tasks. However, the absolute value of human sensitivity to velocity transients depends strongly on the experimental conditions-as a consequence, so does the depth information available from a parallax field evoked by egomotion. Therefore, the question that we ask is: To what extent can we use our high-velocity sensitivity to perform a dynamical control task? With our technique, for the perception of velocity differences, we obtain a Weber ratio that is specific for such a task and that can be compared with data from threshold discrimination experiments. In this way, we will be able to estimate which of those visual field parameters that (according to existing experimental evidence) determine human velocity sensitivity impose perceptual limitations upon real-time navigation tasks.

It is our aim to restrict the parameters that influence the tracking performance to those that are relevant for vision. Therefore, we eliminate sources of mechanical filtering as far as possible. In the experiments, we vary several parameters of the visual field, such as the retinal eccentricity of the center of the stimulating areas and the angular extent of these areas. Under each condition, the subjects conduct the task for a range of velocities. The dynamical properties of the target signal, however, never change. We calculate the cross-correlation between the target signal generated by the computer and the subject's response signal, which is the speed of the pattern to be matched. After compensation for the response delay, we calculate the mean of the modulus amplitude differences between target and response signal and divide it by the mean target velocity in order to obtain a Weber fraction.

\section{METHOD}

\section{Subjects}

Two subjects participated in all experiments: author R.W., male and aged 31, and D.T., male and aged 26. Both were myopes, with corrected-to-normal vision. When the stimulus was projected onto extra foveal positions, care was taken to avoid overlap of blind spot and stimuli. Both subjects were highly trained for the task.

\section{Spatiotemporal Properties of the Stimulus}

The stimulus was displayed within two circular "apertures" on a CRT screen. Through one of the apertures, the subject could see a pattern whose velocity was controlled exclusively by the computer and which, in the sequel, we will refer to as the target patterm. Through the other aperture, the subject could see the pattern that he controlled. We will call this the tracking pattern. These two areas were positioned at variable horizontal distances to the right and to the left of a small mark that the subject fixated while performing the task (Figure 1).

Each pattern consisted of a pseudorandom distribution of bright pixels on a dark background; $25 \%$ of the pixels were white, $75 \%$ were black. Before the onset of an experimental trial, each pattern was stored in a computer frame buffer, a circular part of which was mapped on the screen. The impression of continuous move-
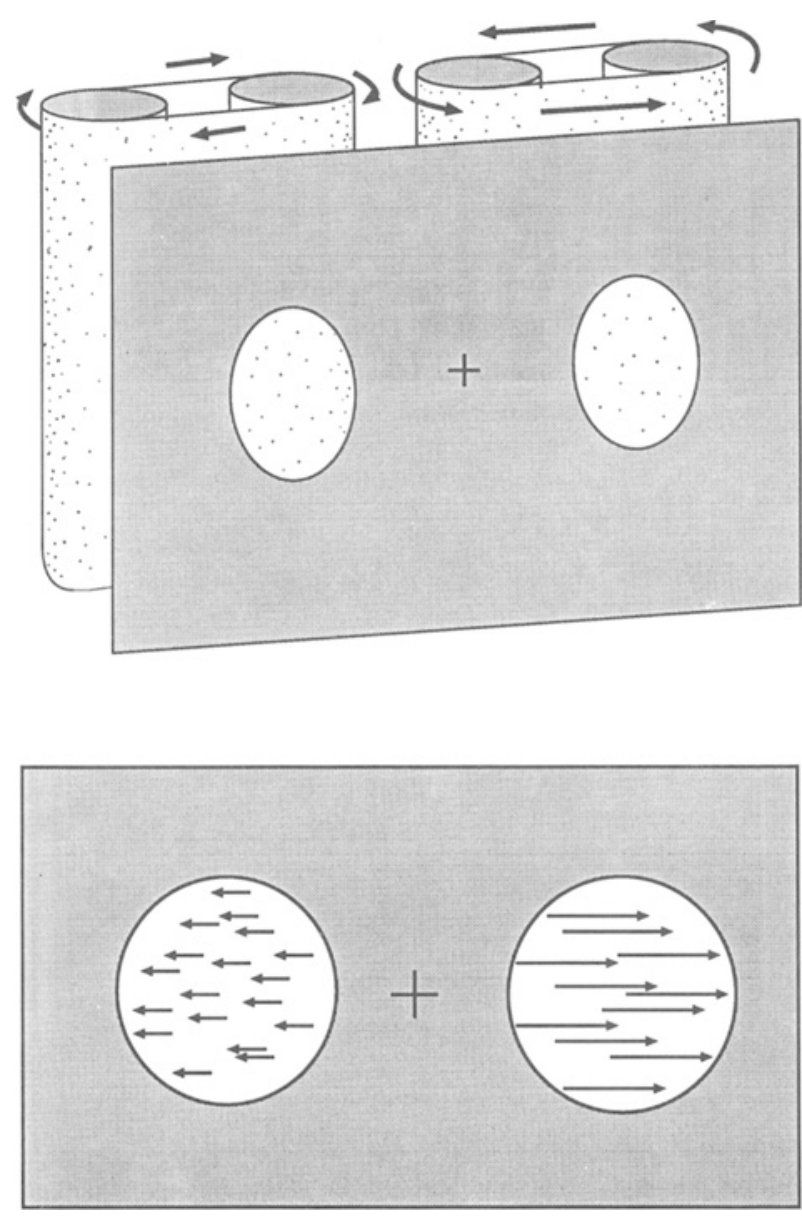

Figure 1. Schematic representation of the stimulus. Two independent frame buffers, each containing a random distribution of white pixels, are shifted relative to the screen. The shifts are of independent speed and in opposite directions. Subjects view each of the moving frames through a separate aperture. The displacement of the patterns resembles the movement of a continuous ribbon behind the screen (see top schema). The subject's task is to control the speed of the pattern seen through the left aperture (symbolized by the short arrows in the bottom schema) such that it matches the speed of the pattern to the right (symbolized by long arrows), which is controlled by a random process.

ment could be evoked by periodically displacing the dot pattern slightly. In principle, this process resembled the movement of a dot pattern printed on a continuous ribbon, with the subject's view partly obscured by a screen containing two circular windows.

The speed of the target pattern varied within an adjustable range in an essentially nonpredictable way. This was achieved by using special noise functions to generate the varying velocities of the target pattern. An experimental trial consisted of 10 sampling sessions, each based on stimulation with a different noise function. These noise functions had equivalent spectral properties. Such a function resulted from summing several sinusoidal functions with identical amplitudes and randomly chosen phase positions. The domain of the noise function's values was then scaled to the adjusted velocity range. In this way, we obtained stimuli with Gaussian-distributed velocities that were nonpredictable. Under all experimental conditions, the same set of 10 noise functions was used to generate the target pattern velocity. The order of the 10 stimulus functions in 
the course of an experimental trial was determined by a pseudorandom selection procedure.

The range of velocities within which the movement of the target pattern varied during a sampling period contained at least 10 discrete steps, where one step was the number of pixels per frame interval. The number of steps increased with the upper limit of the velocity range, which was a parameter.

The pattern velocities were generated by scaling each noise function to the range between zero and the adjustable maximum screen velocity. The mean screen velocity of a single sample was about half the adjusted maximum speed. The mean speeds of the single samples differed slightly from each other, whereas the velocity range was constant in all sampling sessions that represented one experimental condition. For each experimental condition there were three trials, each based on the same set of 10 stimulus noise functions. The velocity range which corresponded to one experimental condition was represented by the mean of the 10 samples. Hence, a parameter value, $\bar{v}$, of about $10 \% / \mathrm{sec}$ mean retinal velocity represented 10 target speed samples, each of which ranged between $0 \% / \mathrm{sec}$ and $20 \% \mathrm{sec}$ angular speed.

To obtain a mean velocity lower than 5 pixels per frame interval, a given pattern position was displayed unchanged in several successive frames. To avoid a change in the number of sample points $(2,000$ for one sampling period), the duration of such a sample was increased. At mean velocities above 5 pixels per frame interval, a sampling interval lasted $56 \mathrm{sec}$ and the shift rate was $35.7 \mathrm{~Hz}$. At a lower mean velocity, the sample duration was $112 \mathrm{sec}$ and the pattern shifted at a rate of $17.8 \mathrm{~Hz}$.

At the lower shift rate of $21.7 \mathrm{~Hz}$, the subjects could perceive that the movement was stroboscopic rather than continuous, although a vivid visual movement was reported in all cases.

\section{Experimental Setup}

During an experiment, the subject sat in a dentist's chair. The back of his head rested against a head support to prevent the subject from changing the distance between the head and the screen. The arms rested on arm supports. Only hand or fingers were used to manipulate the joystick. The stimuli were generated by an Atari Mega ST computer equipped with a blitter chip and were displayed on an Atari SM $12470 \mathrm{~Hz}$ monochrome monitor with a resolution of $640 \times 400$ pixels on a screen area of $13.6 \times 21.7 \mathrm{~cm}$. The mean luminance of the stimulus area was adjusted to about $0.2 \mathrm{~cd} / \mathrm{m}^{2}$. Otherwise, the room was completely dark. The distance between the screen and the subject's eyes was adjusted to 23,50 , or $72.5 \mathrm{~cm}$.

The subject manipulated the speed of the tracking pattern by twisting a specially developed isometric (force-sensitive) joystick about its axis. The joystick was mounted upright at the end of the right arm support.

The task of the subject was to match the quasirandomly varying speed of the target pattern with the speed of the tracking pattern so that symmetrical divergence movement could be perceived.

In order to reduce side effects due to lowpass filtering of hand and fingers (see Results section), the force that had to be applied to the joystick was made independent of the mean speed. This was done by linearly increasing the amplification factor that transformed the output signal of the joystick into the speed of the tracking pattern.

Smaller field sizes were achieved by stepwise increase of the viewing distance between subject and screen. In this way, the number of pixels per stimulus area remained unchanged, while the number of pixels per solid angle as well as the angular size of the individual pixels changed. We made sure that changes in the visible pixel density had no effect on the performance of the task.

When the distance between subject and screen was large (50 or $72.5 \mathrm{~cm}$ ), a second computer monitor was used for stimulation at nonfoveal eccentricities. In these cases, an LED, installed at the corresponding distance, was used as a fixation mark.

\section{Data Evaluation}

In pursuit tracking tasks, the subject's stimulus sensitivity is indicated by the precision with which he or she is able to compensate for the deviation between the target and the tracking signal. In our experiment, these signals were transformed into corresponding pattern speeds. If the subject has access to small signal deviations, the time track of the speed of the tracking pattern will resemble very much the one of the target pattern. If the subject's sensitivity is low, and he/she can react only to large speed deviations, the similarity between the signals will be much lower. The resemblance of signals is mathematically described by the cross-correlation, which is a function of the delay of the subject's reaction. Therefore, we characterize the performance of the subject by calculating the first order maximum of the cross-correlation function for the time track of the target pattern velocity on the one hand and that time track of the target pattern velocity on the one hand and that of the tracking pattern on the other hand. From

$$
R_{v}\left(\Delta t_{k}\right)=\frac{\sum_{i=1}^{N-k}\left[v_{t e}\left(t_{i}\right)-\bar{v}_{t a}\right]\left[v_{t r}\left(t_{i}+\Delta t_{k}\right)-\bar{v}_{t r}\right]}{N \sqrt{\sigma_{v_{t e}} \sigma_{v_{t r}}}},
$$

we obtain the cross-correlation coefficient $R_{y}\left(\Delta t_{k}\right)$ as a function of the time shift $\Delta t_{k}=k \Delta t$ between the two velocity time courses. Here, $\Delta t$ denotes the interframe interval, whereas $v_{t a}, \sigma_{v_{t a}}$ are the velocity and the standard deviation of the target pattern and $v_{t r}, \sigma_{v_{t}}$ are the velocity and standard deviation of the tracking pattern. The parameter $N$ denotes the size of the sample and $i$ the index of the sample time $t$. We define the first order maximum $R_{v}^{*}$ of $R_{v}\left(\Delta t_{k}\right)$ as

$$
R_{v}^{*}:=R_{v}\left(\tau_{0}\right)=\max \left\{R_{v}\left(\Delta t_{k}\right)\right\}
$$

where $\Delta t_{k}<1 \mathrm{sec}$, since for both subjects $\tau_{0}$ always occurs within the first second of an experimental trial (see Figure 2). With this definition, the response delay $\tau_{0}$ is given as the time shift between the two velocity time courses for which the given maximum condition holds. The first order maximum of the cross-correlation of the pattern acceleration $R_{a}^{*}$ is defined in an analogous way. Thus, for one set of 10 time samples, each based on a different stimulating velocity function, one obtains 10 values of first order maxima of the cross-correlation. We calculate the average of the 10 values.

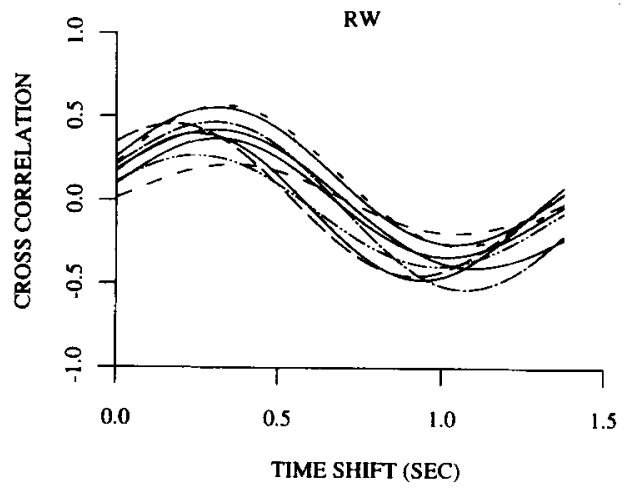

Figure 2. Initial time sequence of the cross-correlation functions of the target and tracking pattern velocity. A data point representing $R_{*}^{*}$ (see text) was obtained by averaging the first order maxima of 10 samples, each of which was based on a different target signal. The target signals differed from each other only whth respect to their phase spectrum. Although the first order peaks of the curves were spread along the time axis, they always lay within the initial 1-sec interval. 
Each experimental trial is repeated three times on different days, and the mean of this $R_{v}^{*}$ triple is presented as a single data point in the following sections.

\section{RESULTS}

\section{Influence of the Statistical Properties of the Speed Modulation}

It is intuitively clear that it is easier to match highly regular variations in the target speed (e.g., sinusoidal changes) than it is to match variations that are considerably irregular. To determine the amount of signal irregularity, which can be considered to be suitable for our experiments, we first want to demonstrate the effect of this nonvisual parameter on the correlation value. The degree of predictability of the target signal is determined by the highest frequency component in the amplitude spectrum of the stimulus noise function. However, while investigating the influence of pattern velocity, stimulus size, and retinal eccentricity, we kept this parameter constant.

Figure 3a shows how $R_{v}^{*}$ and $R_{a}^{*}$ depend on the temporal frequency of the velocity modulation when the target pattern speed is varied sinusoidally. In all situations, the mean retinal velocity is about $15 \%$ sec. Within the range of the lower signal frequencies shown here (up to around $0.75 \mathrm{~Hz}$ ), $R_{v}^{*}$ remains nearly constant whereas $R_{a}^{*}$ increases with frequency until it has reached the level of $R_{v}^{*}$, which is close to maximum. Under the condition of pure sinusoidal stimulation, the signal is completely predictable. After a very short initial period, the subjects are able to adapt to the frequency such that they track the periodically changing pattern velocity with no, or sometimes even with negative, delay. At higher frequencies (above $1 \mathrm{~Hz}$ ), distortions of the response signal lead to lower correlations because the motor system behaves like a lowpass filter (for an introduction to human tracking skills, see Poulton, 1974). At very low frequencies, the tremor in the motor system leads to a strong degradation of the correlation in the second temporal derivative of the pattern's position ( $R_{a}^{*}$ below $0.25 \mathrm{~Hz}$; see Figure $3 a$ ). The situation changes dramatically if the velocity of the target pattern is determined by a noise function. In Figure $3 \mathrm{~b}$, both $R_{v}^{*}$ and $R_{a}^{*}$ are shown as a function of the highest frequency component contained in the stimulating signal. With increasing cutoff frequency, $R_{v}^{*}$ decreases whereas $R_{a}^{*}$ increases initially and then stays at a constant level. This can be interpreted as follows: the more the long-term predictability of the changing pattern velocity decreases due to the number of superimposed higher harmonic components, the more difficult the tracking task becomes (see also Poulton, 1974). At the same time, it seems that, at least at higher frequencies, the subject's ability to detect the polarity in the changing pattern movement (i.e., whether the target pattern is accelerating or decelerating) is not lost.

In the experiments that we describe below, we selected the frequency spectra of the pattern-speed-generating noise function, which had an upper cutoff frequency of $0.702 \mathrm{~Hz}$. With this frequency composition, the predict- (a)

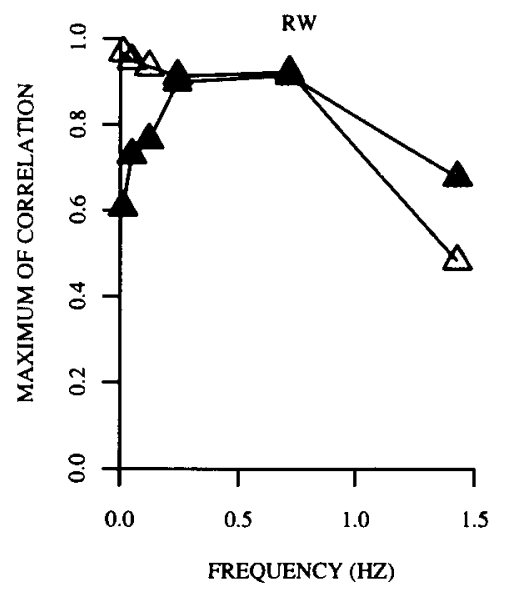

(b)

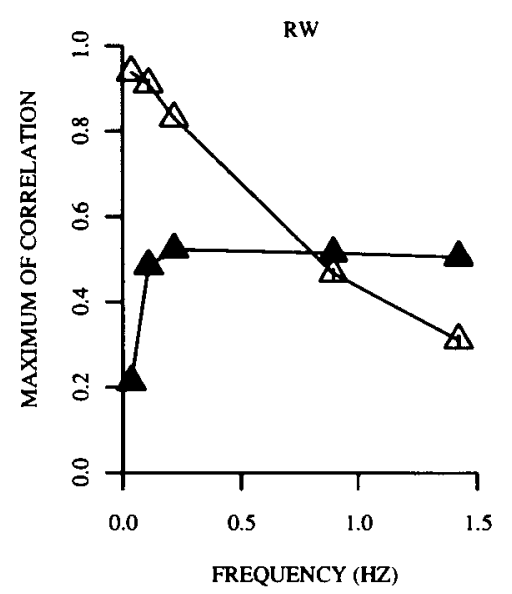

Figure 3. (a) The values of $R_{v}^{*}(\triangle)$ and $R_{a}^{*}(\Delta)$ are plotted against the frequency of a pure harmonic oscillation of the target pattern speed. Above a frequency of around $0.75 \mathrm{~Hz}$, both values decrease. The $R_{v}^{*}$ curve shows the characteristic of a lowpass filter, whereas the $R_{*}^{*}$ curve resembles that of a bandpass filter. In (b) the value on the horizontal axis indicates the highest frequency component of the amplitude spectrum of the function generating the target speed. On the vertical axis, the correlation is again shown. The intersection of the two curves is close to the frequency of $0.702 \mathrm{~Hz}$, which is the highest frequency component of the stimulus functions used in the other experiments.

ability of the stimulus is low enough to ensure that the task is under visual control. Furthermore, this frequency composition has the advantage that both $R_{v}^{*}$ and $R_{a}^{*}$ are at an intermediate level, which, depending on the subject's sensitivity to the specific visual parameters, allows both values to change by the same amount on the correlation scale. At that position in the diagram, the spectrum consists of 26 equally spaced frequency components, ranging from 0.027 to $0.702 \mathrm{~Hz}$.

\section{The Influence of the Field Size}

Figures $4 a-4 c$ (Subject R.W.) and $4 d-4 f$ (Subject D.T.) show the change of $R_{v}^{*}$ with increasing mean velocity of the target pattern when stimulus positions were at different horizontal eccentricities. Each of the diagrams shows 
(a)

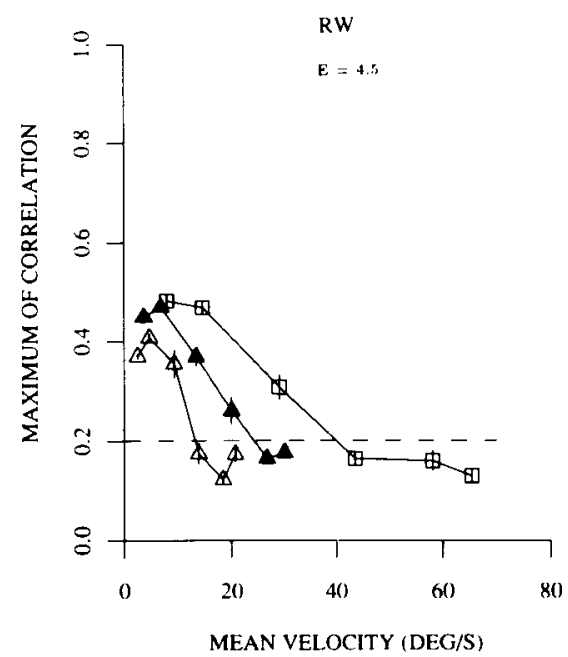

(b)

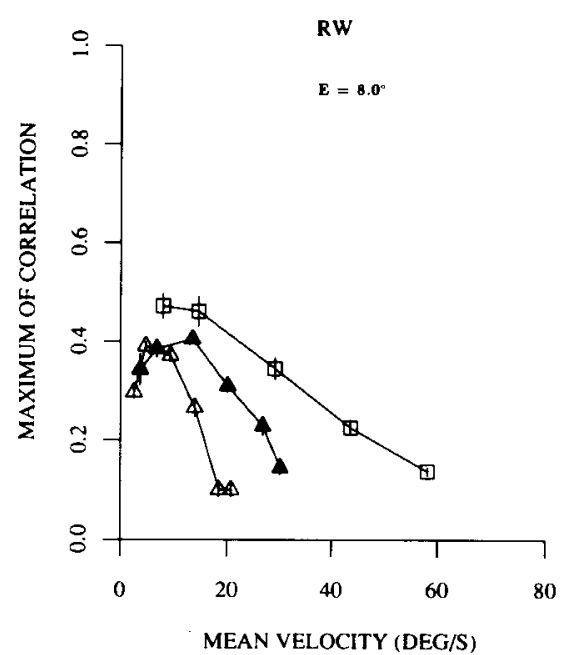

C)

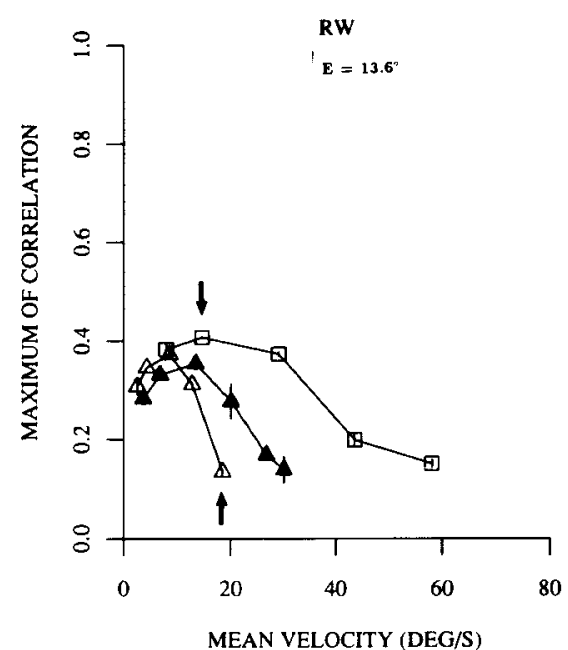

(d)

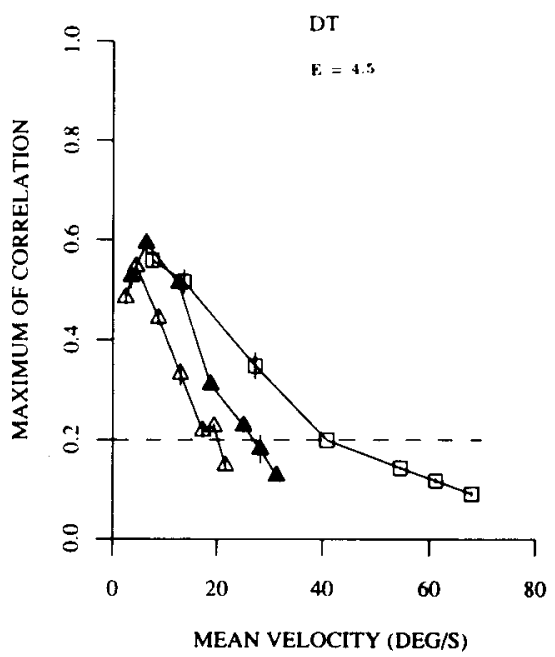

(e)

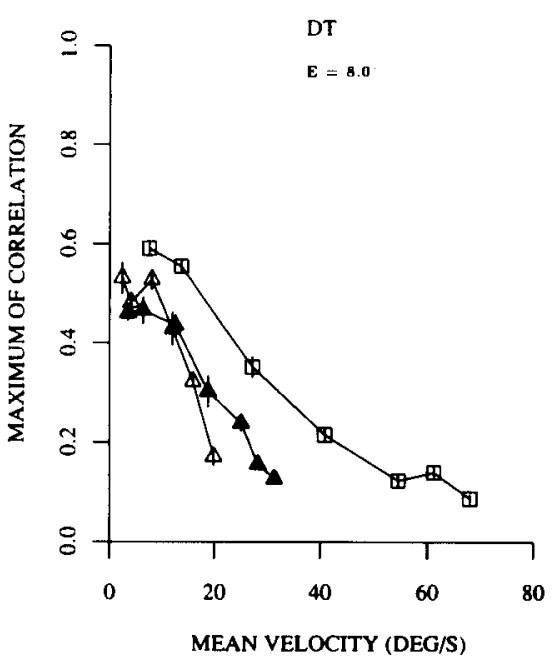

(f)

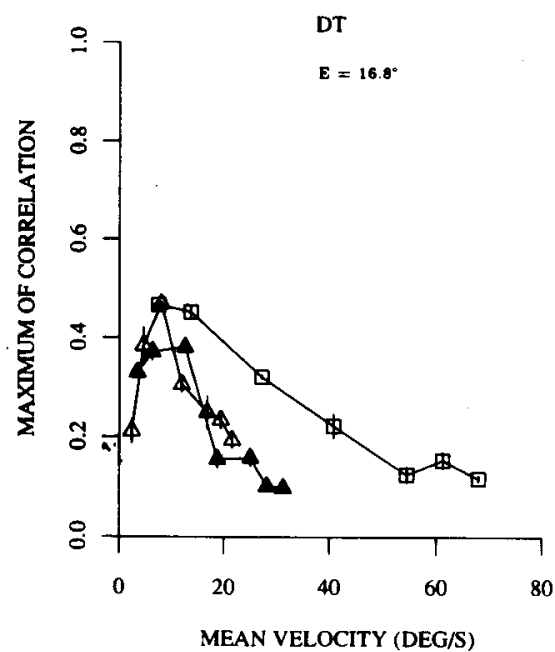

Figure 4. $R_{v}^{*}$ is shown as a function of the mean target velocity. The angular size of the stimulus field is the parameter of the dinferent curves. They have the following field sizes: $3.1^{\circ}(\Delta), 4.6^{\circ}(\Delta)$, and $9.9^{\circ}(D)$. Eccentricities are as given in the diagrams. Vertical bers indicate standard deviations of three measurements. The arrows in (c) point to the data that represent the time tracks in Figure 5 . The dashed line in (a) and (d) indicates an arbitrary threshold performance level (see text for further explanation). 
the results obtained with three different field sizes $\left(3.1^{\circ}\right.$, $4.6^{\circ}$, and $9.9^{\circ}$ of angular extent). In all figures, the vertical bars indicate the standard deviations of three measurements under identical experimental conditions. Figure 4 shows that both subjects performed less efficiently when the average speed was increased. In all cases, after reaching a maximum at rather low retinal velocities, $\boldsymbol{R}_{v}^{*}$ decreased monotonously. However, the position of the maximum and the slope with which $R_{v}^{*}$ decreased depended on the size of the stimulated areas. This observation holds for both subjects.

The only interindividual difference is that the overall performance of Subject D.T. was slightly better. This difference in performance seems to be the same under all conditions.

To obtain an idea of how $R_{v}^{*}$ can be used to compare performances under different conditions, we show in Figure 5 the time courses of the speed of target and tracking pattern for Subject R.W. (Figure 5a, eccentricity $=13.6^{\circ}$, field size $=9.9^{\circ}$; Figure $5 \mathrm{~b}$, eccentricity $=13.6^{\circ}$, field size $=3.1^{\circ}$ ). The response to the same noise functions is shown in both situations. The mean retinal pattern speed is also about the same. The corresponding data points in Figure $4 \mathrm{c}$ are marked with arrows $\left(R_{v}^{*}=0.4\right.$ at field size $9.9^{\circ}$, and $R_{v}^{*}=0.1$ at field size $3.1^{\circ}$ ). The time tracks are plotted in such a way that the subject's reaction, which is delayed by $\tau_{0}$, is compensated by a shift of the tracking pattern with respect to the target pattern. This means that the relative position of the two tracks shown in Figures $5 \mathrm{a}$ and $5 \mathrm{~b}$ is the one that resulted in the maximum value of the cross-correlation function $R_{v}^{*}$ (see Method section).

In Figure 5c, the time tracks of the differences between the target and tracking pattern speeds are plotted together. One can see that the tracking precision is higher in the case of the larger field size. Furthermore, under these conditions, overshoots are mostly followed by an immediate corrective action. This results in oscillation frequencies in the response signal that are not present in the target signal (for the remnant in human tracking performance, see Poulton, 1974). On the other hand, when the field size is small, deviations between target and tracking speed are corrected only when they are fairly large. This explains why the amplitudes of the tracking signal are much higher than those of the target signal. The net effect obtained by averaging the difference signal appears to be stronger in the case shown in Figure 5b. It can be seen, however, that it is not only the decreased sensitivity to the velocity difference due to a reduced stimulus size that expresses itself in the difference signal. The signal oscillations caused by corrective steering actions under the condition of good stimulus visibility show that the subject's strategy also contributes to the difference signal.

We try to quantify the precision of the speed tracking in terms of visual sensitivity to the parameter. If we relate the mean retinal speed difference between the target and tracking pattern (i.e., the integrated time course of the difference signal-such as the one shown in Figure $5 c$-divided by the duration of a trial) to the mean (a)

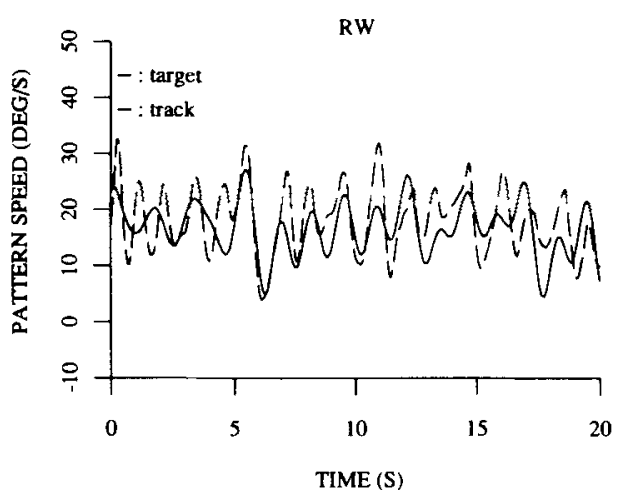

(b)

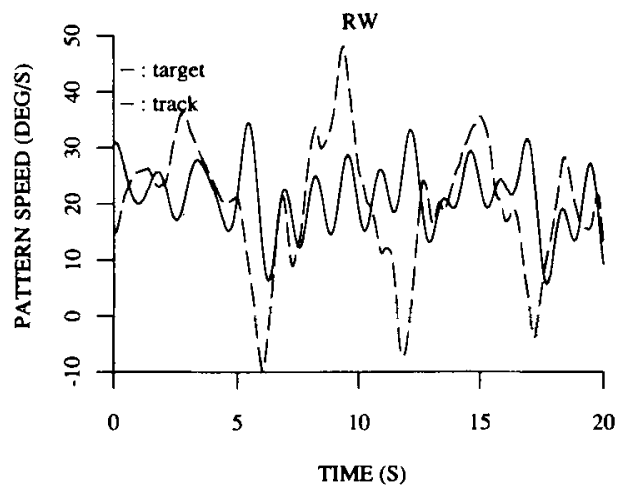

(C)

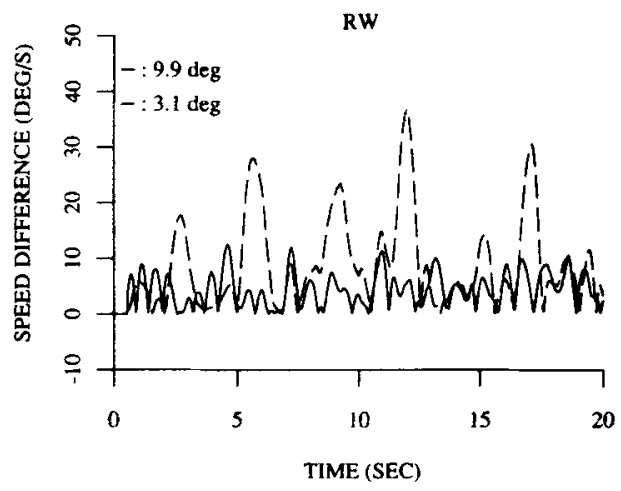

Figure 5. ( $a$ and $b$ ) Parts of the time tracks of the computergenerated target pattern (continuous line) and of the tracking pattern generated by Subject R.W. (broken lines). The time courses of the tracking patterns are shifted to the len by the subject's delay time $\tau_{0}$ (see text for explanation). The eccentricity of the stimulus is $13.6^{\circ}$. The fietd sizes are (a) $9.9^{\circ}$ and (b) $3.1^{\circ}$. The time track of the velocity difference of the curves in (a)-continuous line-and (b)-broken line-is shown in (c).

retinal speed of the target pattern, we obtain the following expression of $W_{v}^{*}$ for the subject's speed sensitivity in the given task:

$$
W_{v}^{*}=\frac{\Delta v_{r m s}(j=k)}{v_{t a}},
$$

where $k$ is the sample index for which $\Delta t_{j}=\tau_{0}$, and where the duration of the trial is expressed by the sample length $N$. 


$$
\Delta v_{r m s}(j)=\sqrt{\frac{\sum_{i=1}^{N-j}\left[v_{t a}\left(t_{i}\right)-v_{t r}\left(t_{i}+\Delta t_{j}\right)\right]^{2}}{N}}
$$

is the root mean square (rms) velocity difference for the time shift $\Delta t_{j}$. Thus, $W_{v}^{*}$ is defined in a way similar to the Weber fraction characterizing the velocity sensitivity measured in psychophysical experiments.

In Figure 6a (Subject R.W., field size $=8^{\circ}$ ) and Figure $6 \mathrm{~b}$ (Subject D.T., field size $=8^{\circ}$ ), $W_{v}^{*}$ is shown for three eccentricities. The $W_{v}^{*}$ values again are the average of three measurements. On the basis of Figure 6 and corresponding data at other eccentricities (not shown here), it appears that the values of $W_{v}^{*}$ obtained with different field sizes coincided as long as the target pattern velocity was low. We pooled all data of one diagram and made

(a)

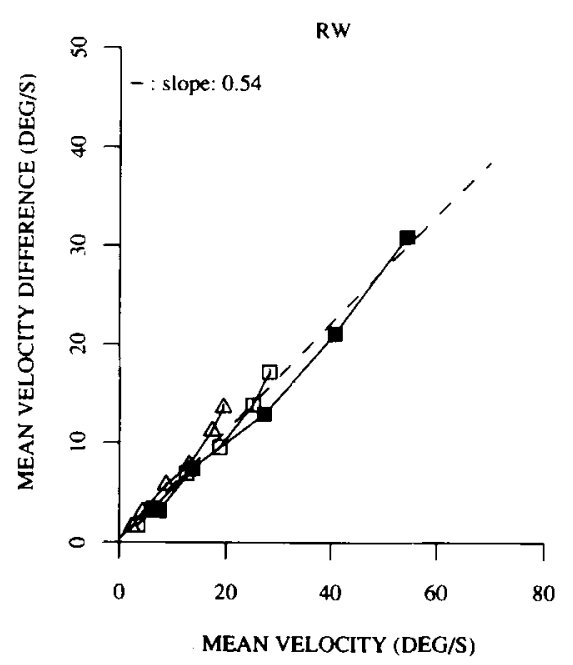

(b)

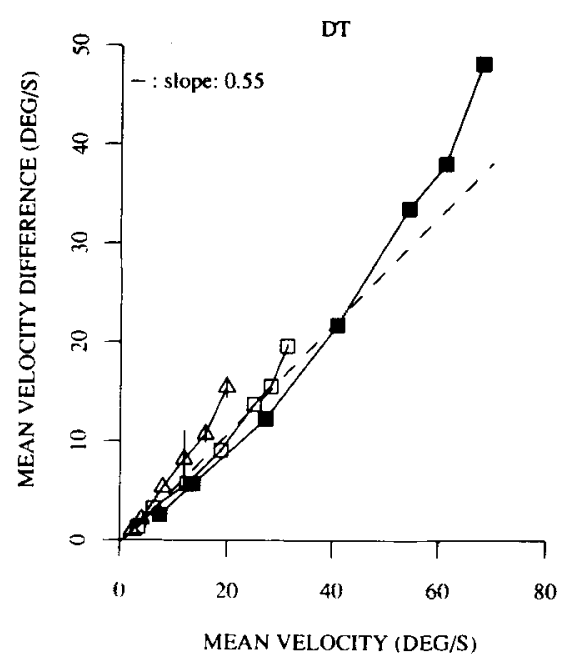

Figure 6. $W^{*}$ is plotted against the mean velocity. The symbols represent the following field sizes: $3.1^{\circ}(\triangle), 4.6^{\circ}(\square)$, and $9.9^{\circ}(\sigma)$. The dashed lines indicate the linear regression of the four lowest W: values of all curves presented in one diagram. (a) Subject R.W. (b) Subject D.T. a linear fit after discarding the two uppermost values of each curve. The regressions are indicated by the dashed lines in Figures $6 \mathrm{a}$ and $6 \mathrm{~b}$. We found a regression coefficient of 0.54 for Subject R.W. and of 0.55 for Subject D.T. The extent of the velocity range within which the data lie close to the regression lines differs with the field size. For small field diameters, the curves start to deviate from the dashed lines at a low velocity. At larger field diameters, the linear range is extended to higher velocities. The linear increase in the velocity difference corresponds fairly well to Weber's law. The velocity range for which this applies, however, appears to be restricted by the size of the stimulus.

\section{The Influence of the Eccentricity}

Figure 7 shows $R_{v}^{*}$ versus the retinal eccentricity of the stimulus position as a parameter rather than the stimulus size (same data as in Figure 4). The latter is constant in each of the plots. At the low velocities, correlation depends on the eccentricity. At higher mean velocities, the curves do not differ significantly. As a tendency in some cases, the position of the maximum of each curve shifts to higher velocities with increasing eccentricity, whereas the magnitude of the maximum correlation decreases with increasing eccentricity. Thus, the optimum speed resolution appears to lie at higher mean velocities when the stimulus position on the retina is more peripheral. The sensitivity to these higher velocities, however, does not exceed the sensitivity to these same velocities when presented at central foveal positions. The effect is diminished at larger field sizes. This can be understood at least partly from the fact that the retinal position is not so sharply localized in these cases. One can say that, within the given range, the influence of the eccentricity on the task performance is much weaker than that of the field size.

The shift of the correlation maxima to higher velocities with increasing eccentricity is more obvious in the time courses of the accelerations of the target and tracking pattern (Figure 8; field size $=3.1^{\circ}$, Subject R.W.). At high velocities, the $R_{a}^{*}$ values increase with eccentricity, whereas, at low velocities, they tend to become lower the more eccentric the stimulus is. This means that at higher pattern velocities the changes in pattern speed become more synchronized when the stimulation is eccentric whereas, at low velocities, this is the case for central stimulation.

\section{DISCUSSION}

\section{The Effect of Stimulus Size}

One of the remarkable features of the curves in Figure 4 is that, at all field sizes, the decrease in correlation is linear within a certain range. From this we can draw some conclusions concerning minimum conditions for the detection of a given velocity. First, we use Figure 4 to estimate the threshold performance, where $R_{v}^{*}$ can be said to be high enough to represent some task performance. In Figures $4 a$ and $4 d$, we chose $R_{v}^{*}=0.2$ as a threshold value and marked it with the dashed line. 
(a)

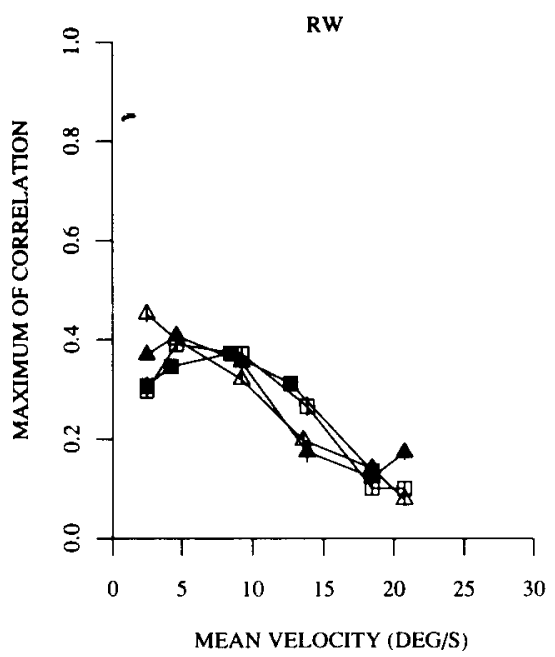

(b)

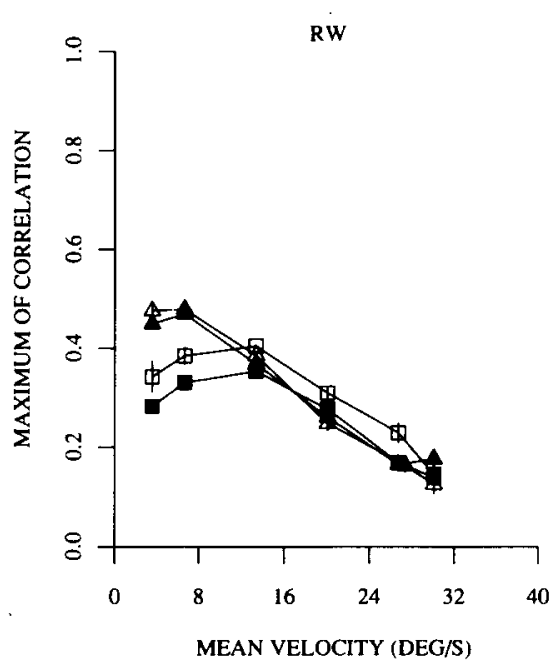

(C)

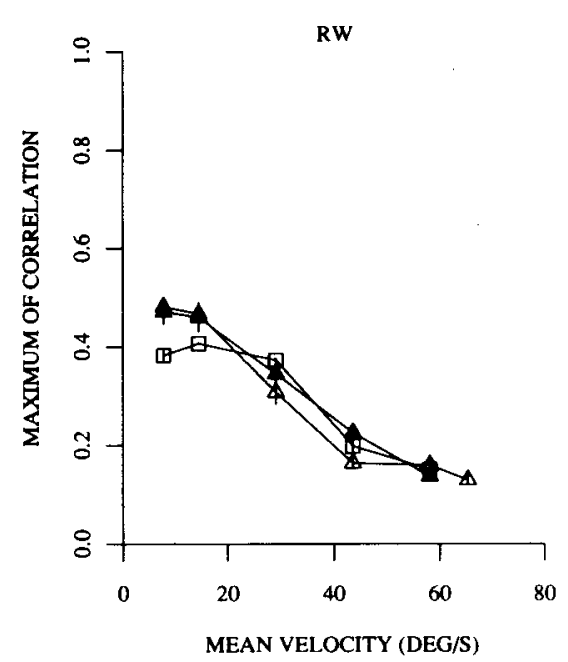

(d)

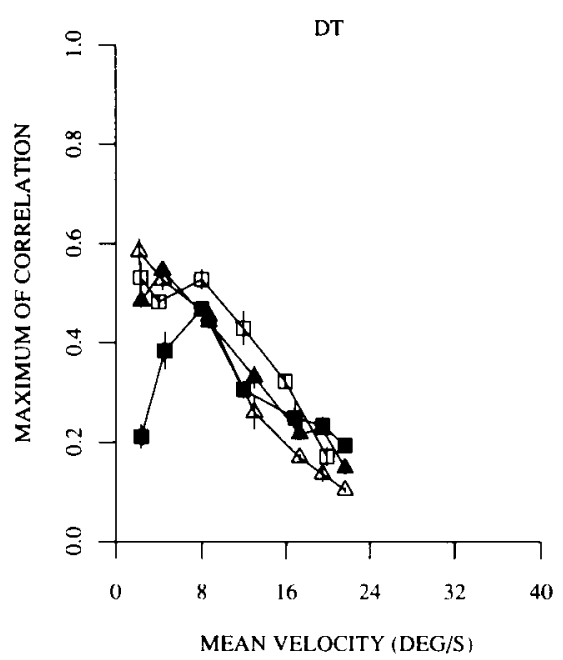

(e)

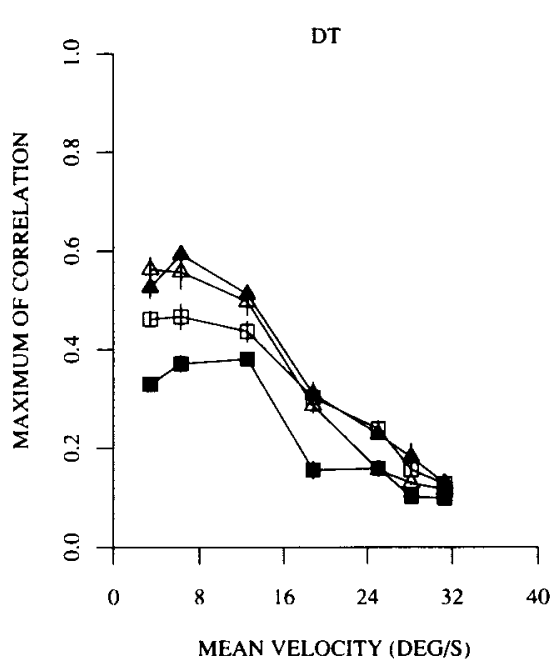

(f)

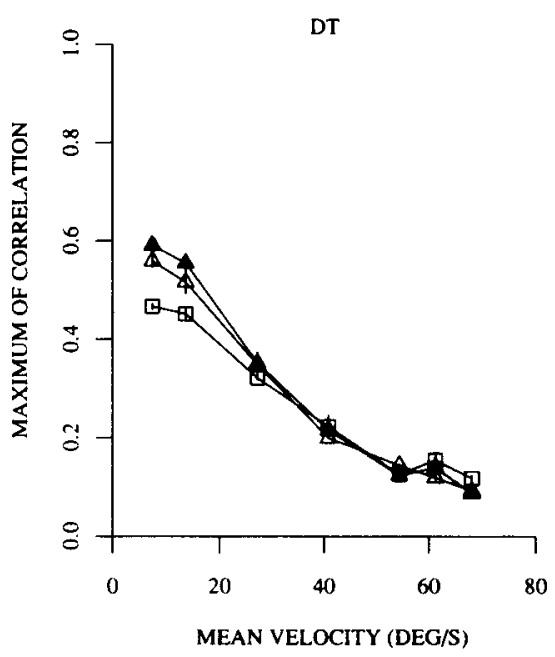

Figure 7. Same data as in Figure 4. Here all data obtained with one field size are presented together in one diagram. The symbols represent different eccentricities: $3.1^{\circ}(\triangle), 4.5^{\circ}(\Delta), 8.0^{\circ}(D) ;(a-c) 13.6^{\circ}$ and $(d-f) 16.9^{\circ}(a)$. Each panel contains data obtained with one field size. For Subject R.W., (a) $3.1^{\circ}$, (b) $4.6^{\circ}$, and (c) $9.9^{\circ}$; for Subject D.T., (d) $3.1^{\circ}$, (e) $4.6^{\circ}$, and (f) $9.9^{\circ}$. 


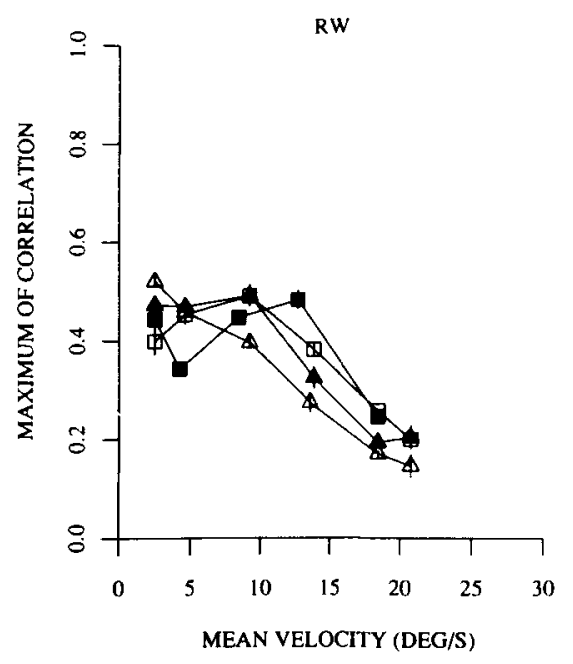

Figure 8. $R_{\text {: }}$ is plotted against the mean stimulus velocity. The symbols represent different eccentricities: $3.1^{\circ}(\Delta), 4.5^{\circ}(\Delta), 8.0^{\circ}$ (ㅁ), and $13.6^{\circ}$ (). With increasing eccentricity, the position of the maximum shifts to a higher mean velocity. Subject R.W.

Figure 9 shows, for both subjects and all eccentricities, the corresponding threshold velocities as a function of the field sizes. The velocities at a given field size lie very close to each other. We conclude from this that the data are invariant with respect to the eccentricity, so all data can be pooled. As an approximation, we fitted a straight line to the data. The slope of this line has the dimension of inverse time. This time can be interpreted as a critical time, $T_{c}(0.2)=D /\left[\bar{v}\left(R_{v}^{*}=0.2\right)\right]$. This is the minimum period of time for which a pattern that moves with mean velocity $\bar{v}\left(R_{v}^{*}=0.2\right)$ has to be visible in the field of width $D$ in order to evoke a threshold velocity percept. At this threshold, the accessible information is just sufficient for the subject to perform the task with the observed minimum correlation of 0.2 . $T_{c}(0.2)$ is about $0.23 \mathrm{sec}$ for Subject R.W. and about $0.29 \mathrm{sec}$ for Subject D.T.

The stroboscopic nature of the pattern displacement sets a theoretical limit on the possibility to perceive the pattern as moving. This limit is given by the pixel shift distance, which is equal to the field diameter. For larger distances, any visible point of one frame is completely uncorrelated with any point of the preceding frame. This maximum distance concerns only those points that really move along the horizontal meridian line of the stimulus area. For all points, traveling along other lines this maximum distance is, of course, shorter. But there is also a physiological reason why one expects this maximum displacement distance to be much shorter than the field diameter. To obtain sufficient noise immunity, several displacement correlations have to be sampled either in parallel or during a time sequence. It has previously been shown by van de Grind, van Doom, and Koenderink (1983) that a minimum number of consecutive displacements of individual points is needed to achieve a percept of coherent movement. This number is more or less fixed for a given subject. Van de Grind et al. report a displacement limit for fast motion detection given by the ratio $D / q$, where $D$ is the field diameter and $q$ is the minimum number of successive frames that contain pixels that can be correlated. The minimum number of displacements is obtained by dividing the time constant $T_{c}(0.2)$, which is independent of eccentricity, and field size by the time interval $\Delta t$ between two frame shifts. It was 8.2 steps for Subject R.W. and 10.3 steps for Subject D.T., whichfor a stimulus width of $3.1^{\circ}$-resulted in $22.3^{\prime}$ and $18.1^{\prime}$ of arc, respectively. This is close to the data presented by van de Grind et al.

(a)

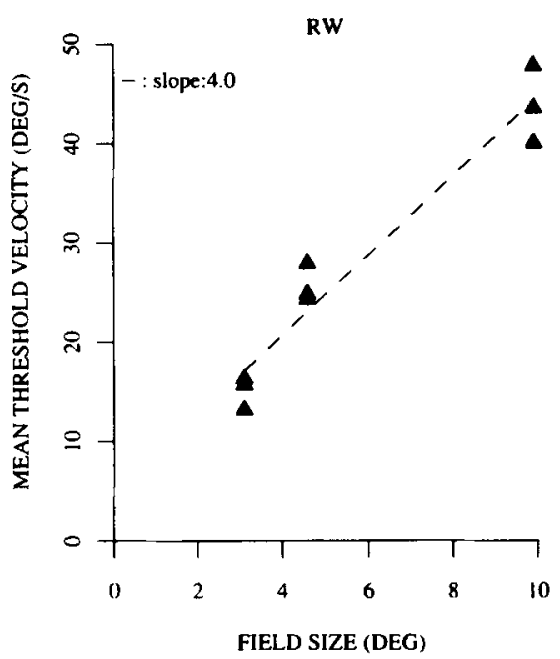

(b)

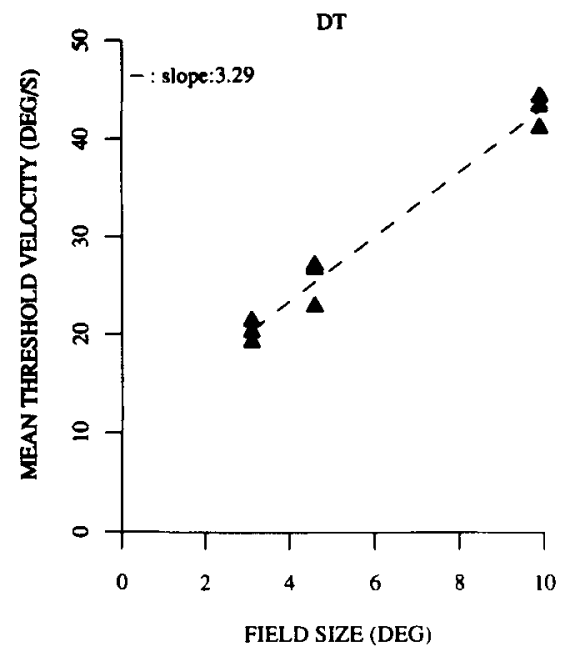

Figure 9. The cutoff values of the mean velocity for which an arbitrary threshold performance has been chosen versus the angular size of the stimulus. All data obtained from 1 subject were pooled for a linear regression. The stopes of the regression lines, 4.0 (Subject R.W.) and 3.29 (Subject D.T.), result in a critical time constant $T_{c}(0.2)$ of 0.23 and 0.29 sec, respectively. 
In conclusion, the less efficient task performance at higher velocities was very probably due to a reduced velocity percept. The velocity range for which we found a significant performance was limited primarily by the size of the stimulated retinal area. We can assume that the range within which $W_{v}^{*}$ corresponds to Weber's law is limited by the lower and upper threshold for velocity detection. The velocities of the hands of a watch, for example, cannot be discriminated because both are below the lower threshold, whereas, above the upper threshold, everything looks blurred. That this upper threshold is dependent on the stimulus field size can be seen from the data in Figure 6, where the points at the highest mean velocities presented at a given field size start to deviate from straight lines.

\section{General Remarks}

We described an experiment in which single parameters of the visual field, which determine human sensitivity to differential motion, can be varied in isolation while others are kept constant. Besides the spatial and temporal gradient in the velocity field, we should mention at least two other important parameters of the optic flow-the angular size of the visible part of the surrounding scene and its position in the visual field. In situations where vehicles have to be controlled, the angular size and location of the stimulus in the visual field are determined mostly by the design of the windscreen. To ensure that our results concerning the influence of these parameters on velocity detection are of any relevance for natural navigation tasks (e.g., car driving or flying an aircraft, etc.), we resorted to a dynamical tracking technique. It is known from threshold discrimination experiments (Koenderink, van Doorn, \& van de Grind, 1985; McKee \& Nakayama, 1984; van de Grind et al., 1983) that the angular extent as well as the eccentricity of the stimulated area on the retina are parameters that play a critical role for the determination of visual velocity sensitivity. Since the sensitivity is suprisingly high under certain conditions, the question arises whether limiting factors still are of any influence or whether the experimental conditions are based on dynamical speed discrimination rather than on discrimination between discrete instantaneously presented stimuli. We regard the dynamic aspect of our discrimination task to be the crucial condition that makes the experiment a reasonable representation of real-time navigation tasks. Our data prove to be comparable to those of other reports about visual sensitivity to moving patterns (van Doorn \& Koenderink, 1982). Over a broad range of varying parameters, we found a Weber ratio of around 0.55 for the dynamic control of differential speed.

The range over which the sensitivity corresponds to Weber's law is restricted by an upper velocity cutoff, which is field-size dependent. This is in agreement with the results of Koenderink et al. (1985), who found that, above a retinal stimulus velocity of about $20 \% \mathrm{sec}$, the ability to segregate parts of the visual field on the basis of velocity transients is limited by the width of the stimulus field in the direction of the movement. We find that a pixel that travels through an aperture has to be visible, on average, for a minimum time interval, $T_{c}$, in order to evoke an impression of coherent movement sufficient to perform the task described. This time interval is independent of the field size (see Figure 9). If one considers the shift rate of the stimulus, this constant time interval is equivalent to the minimum number of pixel displacements necessary to detect the velocity at the field-size-dependent cutoff value. Such a minimum number of pixels, which at least $\mathrm{h}$ is to be correlated to perceive coherent movement, has also been reported by van de Grind et al. (1983). These authurs interpreted this observation in terms of a minimum condition for temporal sampling of the given velocity that ensures sufficient noise immunity in order to perceive the velocity. Introducing a threshold performance by means of a saturation level of the correlation value enables us to reproduce this minimum condition for speed resolution with our approach.

The delay time $\tau_{0}$ of the subject's response was typically between 0.3 and $0.5 \mathrm{sec}$ (see Figure 2). A slight increase of $\tau_{0}$ (up to maximum $0.8 \mathrm{sec}$ ) was only observed when the correlation was near the threshold value of about 0.2 . The value of $\tau_{0}$ is determined primarily by the degree of predictability of the stimulus. If mechanical delay can be avoided, the special layout of the tracking task seems to be of minor importance for the performance level. Concerning an experiment in which a light source that moved pseudorandomly on a rail in one dimension had to be tracked, van der Meulen (1989) reported a delay of aiout 250-300 msec. However, the stimulus functions he used contained only seven harmonic components and the subjects had to make real arm movements. For the tracking of pseudorandomly moving targets with the eye, typical delay times are $125 \mathrm{msec}$ for the smooth pursuit system (Robinson, 1965; Westheimer, 1954a) and $200 \mathrm{msec}$ for the saccadic system (Stark, Vossins, \& Young, 1962; Westheimer, 1954b).

Within the range of retinal positions that we studied, we are not able to demonstrate clearly that the stimulus eccentricity influences the tracking performance. A small effect, however, can be seen in Figures $7 \mathrm{a}$ and $7 \mathrm{~b}$, which show the finest resolution of the eccentricity influence (stimulus field size $=3.1^{\circ}$ ). We found that, for both subjects, the mean velocity of about $10 \% / \mathrm{sec}$ could be resolved equally well at all eccentricities, whereas lower velocities could be sufficiently resolved only at rather central stimulus locations.

With regard to the synchronization of the subject's tracking actions with the changing stimulus speed, the intersection of the curves in Figure 8 shows that it is only at a central stimulus location that the low-velocity resolution is sufficient to achieve higher correlations of the acceleration signals. At higher velocities, the timing of decelaration and acceleration is somewhat better when the stimulus is viewed more eccentrically. This might be an indication for a peripheral specialization for the detection of accelerating targets. This could be of special use for 
solving more complex navigation tasks than the one we described in this paper.

The experimental procedure is suitable for establishing a link between psychophysics and visually guided control tasks that occur in daily situations. It provides a tool to judge how far implications of psychophysical data obtained under optimal perceptual conditions impose critical constraints for practical tasks.

\section{REFERENCES}

De Bruyn, B., Orban, G. A. (1988). Human velocity and direction discrimination measured with random dot patterns. Vision Research, 28, 1323-1335.

GiBson, J. J. (1950). The perception of the visual world. Boston, MA: Houghton Mifflin.

Koenderink, J. J., van Doorn, A. J., a van DE Grind, W. A. (1985). Spatial and temporal parameters of motion detection in the peripheral visual field. Joumal of the Optical Society of America A, 2, 252-259.

MCKEE, S. P. (1981). A local mechanism for differential velocity detection. Vision Research, 21, 491-500.

McKee, S. P., \& Nakayama, K. (1984). The detection of motion in the peripheral visual field. Vision Research, 24, 25-32.

NAKAYAMA, K. (1981). Differential motion hyperacuity under conditions of common image motion. Vision Research, 21, 1475-1482.
Poulton, E. C. (1974). Tracking skill and manual control. New York: Academic Press.

Robinson, D. A. (1965). The mechanisms of human smooth pursuit eye movement. Journal of Physiology (London), 180, 569-591.

Rogers, B., Graham, M. (1979). Motion parallax as an independent cue for depth perception. Perception, 8, 125-134.

Stark, L., Vossins, G., Young, L. R. (1962). Predictive control of eye tracking movements. IEEE Transactions on Human Factors in Electronics, 3, 53-57.

VAN DE Grind, W. A., van Doorn, A. J., Koenderink, J. J. (1983). Detection of coherent movement in peripherally viewed random-dot patterns. Joumal of the Optical Sociery of America A, 73, 1674-1683.

VAN DER MEULEN, J. H. (1989). Viswomotor performance in childhood: A study on development and dysfunction. Doctoral thesis, University of Utrecht, The Netherlands.

VAN DOORN, A. J., Koenderink, J. J. (1982). Visibility of movement gradients. Biological Cybermetics, 41, 167-175.

VAN DoORN, A. J., \& Koenderink, J. J. (1983). Detectability of velocity gradients in moving random-dot patterns. Vision Research, 23, 799-804.

Westheimer, G. (1954a). Eye movement responses to a horizontally moving visual stimulus. Archives of Ophthalmology, 52, 932-941.

Westheimer, G. (1954b). Mechanisms of saccadic eye movements. Archives of Ophthalmology, 52, 710-724.

(Manuscript received February 15, 1991; revision accepted for publication December 19, 1991.)

\section{Call for Assistance in The Compilation of a History of the Psychonomic Society}

The Governing Board of the Psychonomic Society is pleased to announce that Robert C. Bolles has agreed to serve as the first Historian of the Society.

All members who might have information relevant to this undertaking are invited to send it directly to Dr. Bolles. Founding members and those who attended the early meetings are especially encouraged to record their reminiscences. While Dr. Bolles hopes to collect as much information as possible relevant to the history of the Society, he will concentrate first on the early history.

Dr. Bolles's address is Department of Psychology, University of Washington, Seattle, Washington 98195 (phone: 206-543-2631). 